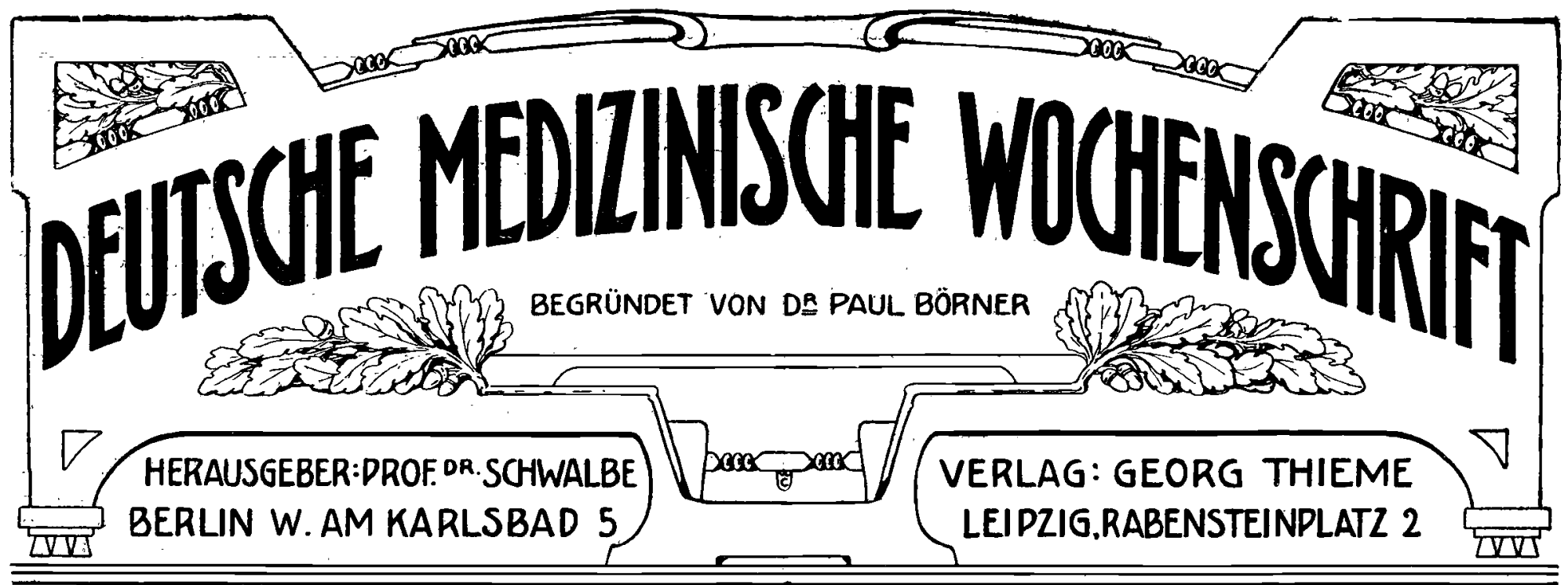

Aus der I. Medizinischen Klinik der Universität in Berlin. (Direktor: Geh. Med.-Rat Prof. Dr. His.)

\section{Ueber Verlangsamung des Stoffwechsels.')} Von R. Staehelin.

Vor einigen Jahrzehnten war viel von Verlangsamung des Stoffwechsels die Rede. Seit genaue Stoffwechselversuche in größerer Zahl angestellt worden sind, hat sich gezeigt, daß die meisten Stoffwechselvorgänge auch in Krankheiten viel konstanter sind als man angenommen hatte, und es ist heutzutage ganz ,unmodern, von Verlangsamung des Stoffwechsels zu sprechen. Nun aber beginnen sich die Anzeichen dafür zu mehren, daß dieser Begriff wieder zu Ehren gelangen könnte. Ich möchte mir deshalb erlauben, die verschiedenen vorliegenden Beobachtungen zusammenzufassen, und ich hoffe, Ihnen zeigen zu können, daß sowohl eine Verlangsamung einzelner Stoffwechselprozesse, als auch eine „Verlangsamung “ der gesamten. Oxydationsvorgänge, eine Herabsetzung des Energieverbrauchs, in der Pathologie eine größere Rolle spielen, als heute, vielfach angenommen wird.

Wenn wir zunächst den Stoffwechsel einzelner Substanzen betrachten, so sehen wir, daß schon normalerweise die Zersetzung eines Stoffes verlangsamt werden kann, wenn eine Nahrung zugeführt wird, deren Verbrennung den anderen Stoff aus der Zersetzung verdrängt. Gibt man Eiweiß zusarnmen mit Kohlehydrat oder Fett, so erscheint der Stickstoff viel langsamer im Urin, als wenn man die gleiche Menge Eiweiß allein verabreicht. Eine gleiche Verlangsamung der Eiweifzersetzung, wie sie beim Gesunden durch Zufuhr eines anderen Stoffes herbeigeführt wird, kann beim Kranken durch den pathologischen Prozeß selbst bewirkt werden. Das scheint beim Diabetes der Fall zu sein. Falta und Gigon²) fanden bei Zuckerkranken, wenn sie während einer Periode konstanter Kost an einem Tage eine größere Menge Kasein der Nahrung zulegten, eine deutlich verlangsamte Ausscheidung des superponierten Stickstoffs gegenüber dem Gesunden. Sie weisen auch auf die schon vor 40 Jahren von $\mathrm{Koch}^{3}$ ) gefundene Tatsache hin, daß beim Diabetiker die Hauptmenge des Harnstoffes nicht bei Tage, sondern erst in der Nacht ausgeschieden wird.

Viel ausgesprochener fand ich die Verlangsamung der Eiweißzẹrsetzung in einem Fall von Fettsucht.4)

Der $83,5 \mathrm{~kg}$ schwere Patient erhielt eine ganz genau konstante Kost. Am neunten Tage wurden außerdem $80 \mathrm{~g}$ Kasein verabreicht. Während nun beim Gesunden die N-Ausscheidung bei dieser Versuchsanordnung sofort rasch in die Höhe geht und bald wieder absinkt, sodaß am dritten Tage fast ebensoviel $N$ wieder ausgeschieden ist, wie eingeführt wurde, stieg bei diesem Fettsüchtigen die N-Ausscheidung am ersten Tage nur wenig an und sank am zweiten schon

1) Vortrag, gehalten im Verein für innere Medizin in Berlin am l. Mărz 1909. - 2). Ueber die Gesetze der Zuckerausscheidung beim Diabetes mellitus, 5. Mitteilung. Zeftschrift für klinische Medizin, Bd. $65 .-3$ ) Ueber Diabetes mellitus. Inaug.-Diss. Jena 1867 . - 4) $\mathrm{Staehel1 \textrm {n } \text { , Der respiratorische Stoffwechsel eines Fettsúchtigen. }}$ Zeitschrift für klinische Medizin, Bd. 65 . wieder sehr tief, sodaß an den drei Tagen nur etwa halb so viel $\mathrm{N}$ ausgeschieden wurde, als mit dem Kasein zugeführt war.

Ich fand auch, ähnlich wie Jaquet und Svenson, nach Eiweißnahrung eine auffallend lang hingezogene Erhöhung des Sauerstoffverbrauchs. $\mathrm{Zu}$ einem abschließenden Urteil wird man freilich erst gelangen können, wenn mehr Versuche sowohl an Kranken als auch an Gesunden vorliegen.

Viel besser untersucht ist der zeitliche Ablauf der Purinausscheidung. Ich kann mich hier kurz fassen, da der Vortrag von Herrn Brugs ch und die anschließende Diskussion ${ }^{1}$ ) sich ausführlich damit beschäftigt haben. Die einfachste Erklärung der Abnormitäten, die die Purinausscheidung bei der Gicht zeigt, ist doch wohl die Annahme eines verlangsamten Nukleïnstoff wechsels bei dieser Krankheit.

Ein ähnliches Verhalten der Harnsäureausscheidung wie bei der Gicht, also wohl auch eine Verlangsamung des Nukleïnstoffwechsels, findet sich auch bei Bleivergiftung, bei Potatoren und bei einzelnen Fällen von Diabetes, wie Bloch ${ }^{2}$ ) gezeigt hat. Auf die Zuckerkrankheit möchte ich besonders hinweisen, da ja, wie wir gesehen haben, auch eine Verlangsamung des Eiweißstoffwechsels bei ihr vorzukommen scheint.

Während Eiweif und Nukleïnsubstanzen leicht bestimmbare Endprodukte liefern, die den zeitlichen Ablauf der Zersetzung zu verfolgen erlauben, ist das bei Kohlehydraten und Fetten nicht der Fall. Doch kann uns wenigstens für den Kohlehydratstoffwechsel der respiratorische Quotient Anhaltspunkte bieten. Mohra ${ }^{3}$ hat aus seinem Verhalten nach Fleischnahrung geschlossen, daß im Diabetes mellitus die Zuckerverbrennung verlangsamt sei. Diese Hypothese ist recht einleuchtend. Wenn das Vermögen des Organismus, Zucker zu verbrennen, herabgesetzt ist, so erfolgt die Verbrennung auch langsamer. Doch muß bemerkt werden, daß durch die Mohrschen Versuche ein langsamerer Ablauf der Zuckerverbrennung nicht bewiesen ist.

Beim Diabetes würde eine Verlangsamung der Verbrennung nach dieser Anschauung mit einer unvollständigen Verbrennung vorbunden sein, doch läßt sich auch denken, daß in anderen pathologischen Zuständen die Verbrennung der Kohlehydrate zwar langsam, aber doch vollständig erfolgt. Das scheint bei Fett. süchtigen der Fall zu sein. Bei einem von mir untersuchten Patienten ${ }^{4}$ ) fand ich mehrmals noch nach 12 stündigem Hunger auffallend hohe respiratorische Quotienten, und auch in manchen Versuchen anderer Autoren läßt sich diese Beobachtung machen. Endlich hat $\mathrm{Pari}^{5}$ ) aus dem Ablauf der Stickstoffausscheidung bei thyreoidektomierten Hunden geschlossen, daß bei Ausfall der Schilddrüsenfunktion der Kohlehydratstoffwechsel verlangsamt ist.

Noch weniger als über den zeitlichen Ablauf der Kohlehydratverbrennung wissen wir über den der Fettzersetzung.

1) Siehe diese Wochenschrift 1908, No. 51 und 1909, No. 1. - 2) Bloch, Deut sches Archiv für klinische Medizin, Bd. 83. - 3) Mohr, Zeitschrift für experimentelle Pches Archiv für klinische Medizin, Bd. 83. - 3) M o h r, Zeitschrift für experimentelle Pathologie und Therapie, Bd. 4. - 4)
chemische Zeitschrift, Bd. 13, S. 281. 
Waldvogel ${ }^{1}$ ) glaubt zwar eine Verlangsamung eines intermediären Prozesses, der Azetonbildung aus $\beta$-Oxybuttersäure, bei Fettsüchtigen gefunden $\mathrm{zu}$ haben. Leider sind seine Resultate bisher noch nicht nachgeprüft worden.

Eine Verlangsamung der Fettverbrennung läßt sich aber kaum denken ohne eine Herabsetzung des gesamten Energieverbrauchs. Eine solche bereitet nun den herrschenden Anschauungen eine gewisse Schwierigkeit. Bei zunehmender Außentemperatur nimmt die Wärmeproduktion des Warmblüters, auch im Ruhe- und Hungerzustand, immer mehr ab; diese Abnahme macht aber an einem bestimmten Punkte Halt, und die weitere Erhöhung der Umgebungstemperatur vermindert die Wärmeproduktion nicht mehr, die Wärmeproduktion bleibt unabhängig von der Außentemperatur. Die nächstliegende Annahme ist die, daß in diesem Zustand der physikalischen Wärmeregulation der Stoffwechsel auf das Minimum reduziert ist, bei dem die Erfüllung der geforderten Leistungen noch möglich ist, also bei Körperruhe und im Hunger die kleinste Größe, bei der die Organfunktionen ungestört aufrecht erhalten werden können. Rubner sagt:

"Gerade der Umstand, daß das Tier bei hoher Temperatur auf einem Minimum des Kraftwechsels anlangt, zeigt die innere Not wendigkeit des Verbrauches, . und dieser ist auch der Stimulus, welcher die Grenze der Leistungen nicht sinken läßt".

Es erscheint uns daher kaum wahrscheinlich, dab der Energieverbrauch erheblich vermindert werden könnte, ohne dab schwere allgemeine Schädigungen des Körpers auftreten.

In dem einen pathologischen Zustand, in dem eine stärkere Herabsetzung des Energieverbrauchs allgemein anerkannt ist, dem Ausfall der Schilddrüsenfunktion, finden wir solche allgemeine Schädigungen sehr ausgesprochen. Meist sind die Ausfallserscheinungen somatischer und psychischer Natur. In dieser Hinsicht ist nun ein Fall von Cachexia strumipriva, den ich zusammen mit $\mathrm{Hagenbach}$ und $\mathrm{Nager}$ untersucht habe, ${ }^{2}$ ) nicht chne Interesse.

Der Junge hatte mit 12 Jahren nach operativer Entfernung eines Kropfes zu wachsen aufgehört, war sehr mager und hatte einen äußerst geringen Appetit. Der Sauerstoffverbrauch in 12 Stunden während Bettruhe betrug nur. $70 \%$ dessen, was ein gleich groß3es und gleich schweres (jüngeres) Kind konsumierte. Schilddrüsenmedikation erhøhte den Sauerstoffverbrauch in vier Wochen un 25\%. Das Interessanteste dieses Falles besteht nun darin, daß der Knabe psychisch ganz normal war und keinerlei Intelligenzdefekte aufwies, auch kơrperlich im Verhältnis zu seinem Ernährungszustand recht leistungsfähig war.

Diese Beobachtung zeigt, daß eine erhebliche Einschränkung der Wärmeproduktion bei relativ geringen anderweitigen Ausfallserscheinungen möglich ist, und wir dürfen deshalb die Möglichkeit einer Herabsetzung des Energieumsatzes bei Kranken und unter Umständen auch bei Gesunden nicht ohne weiteres ablehnen.

Betrachten wir zunächst das Vorkommen einer Herabsetzung des Energieverbrauchs bei Gesunden. Den Aerzten ist es schon lange aufgefallen, daß viele Menschen dann, wenn sie in ihrem Ernährungszustand stark reduziert sind, oft mit fabelhaft geringen Nahrungsmengen auskommen, ohne an Gewicht abzunehmen. Schon Pettenk ofer und Voit ${ }^{3}$ ) haben in einem solchen Fall eine Herabsetzung der Kohlensäureproduktion nachgewiesen; Fr. Müller und Neb elthau haben Fälle genau beobachtet, in denen durch Verätzung des Oesophagus die Speiseaufnahme beschränkt war und sich der Körper an die geringe Nahrungsaufnahme durch Herabsetzung des Energieumsatzes angepaßt hatte.

Besonders schön ist die Untersuchung Magnus-Levys s${ }^{4}$ ) an einem Patienten, der durch freiwillige Inanition auf $36 \mathrm{~kg}$ abgemagert war und dabei einen fabelhaft niedrigen Sauerstoffverbrauch aufwies. Sobald er normale Nahrungsmengen erhielt, wurde der Sauerstoffkonsum normal.

Aber nicht nur in diesen Fällen extremer Inanition, die schon beinahe in das Gebiet des Pathologischen gehören,

1) Deutsches Archiv für klinische Medizin, Bd. 89. - 2) Noch nicht veröffentlicht. -3) Literatur s. v. Noorden, Handbucl der Pathologie des Stoffwechsels. 2. Aufl. Bd. 2, S. 480 ff. v. B e r g man n, Zeitschrift für experimentelle Pathologie und Therapie Bd. 5. - 4) Der Einflus von Krankheiten auf den Energiehaushalt im Ruhezustand. Zeitschrift für klinische Medizin, Bd. 60 kommt eine Anpassung des Verbrauchs an die Zufuhr vor. R. 0. Neumann wies in monate- und jahrelang fortgesetzten mühsamen Selbstversuchen nach, daf er bei gleichen körperlichen Leistungen sein Körpergewicht zu verschiedenen Zeiten mit ganz verschiedenen Nahrungsmengen aufrecht erhalten konnte. Aus einzelnen der Versuche Chittendens geht endlich mit Sicherheit hervor, daß eine Reduktion speziell der Eiweißzufuhr eine Verminderung des Nahrungsbedürfnisses zur Folge haben kann.

Auch in Tierversuchen läßt sich bisweilen eine solche Anpassung nachweisen, worauf $\mathrm{Rubner}^{1}$ ) ausdrücklich hinweist. Falta, Grote und ich $^{2}$ ) konnten an einem Hunde dieselbe Beobachtung machen.

Wir wissen aber noch nicht, wie häufig diese Fähigkeit, den Stoffumsatz bei verminderter Zufuhr einzuschränken, vorkommt. Daß von den Hunden nur ein Teil dieses Verhalten zeigt, hat schon Rubner betont. Beim Menschen scheint es noch seltener zu sein. Die meisten verhalten sich wie der von Caspari untersuchte Rohkostvegetarier, ${ }^{3}$ ) der trotz höchstgradiger Unterernährung keinerlei Einschränkung des Energieumsatzes erkennen ließ.

Bei der Beurteilung der krankhaften Zustände, in denen eine solche Herabsetzung des Energieverbrauchs in Frage kommt, müssen wir uns an diese schon beim Gesunden vorkommenden individuellen Verschiedenheiten erinnern. Tatsächlich finden wir auch, wie Svenson gezeigt hat, daß in der Rek onvaleszenz von konsumierenden Krankheiten einzelne Patienten einen herabgesetzten Sauerstoffverbrauch aufweisen, andere nicht. Wo diese Herabsetzung vorkommt, hält sie nur sehr kurze Zeit an, weil der nun beginnende Eiweißansatz die Wärmeproduktion mächtig steigert.

Etwas weniger klar liegen die Verhältnisse beim Diabetes mellitus. Weitaus die meisten Zuckerkranken haben einen normalen oder sogar einen etwas vermehrten Sauerstoffverbrauch. Es gibt aber einzelne Kranke, die bei einer starken Reduktion der Nahrungsmenge nicht, wie man erwarten sollte, abnehmen, sondern ihr Körpergewicht beibehalten. v. Noorden meint, daß alle die Fälle in die eben erwähnte Kategorie von unterernährten Individuen gehören. Das scheint mir aber nicht alles zu erklären. So habe ich einen Patienten mit schwerem Diabetes beobachtet, der sein Körpergewicht von $67 \mathrm{~kg}$ bei einer nutzbaren Energiezufuhr von weniger als 30 Kalorien pro Kilogramm aufrecht erlielt, trotzdem er sich viel in Zimmer bewegte. Das würde eine Herabsetzung der Wärmeproduktion von etwa $10 \%$ bedeuten. Ich gebe zu, daf eine solche Beobachtung noch nicht beweisend ist. Man hat aber vielleicht bisher zu sehr nach exorbitant hohen Veränderungen des Energiehaushalts gesucht, sodaß solche Fälle vielleicht häufiger sind, als angenommen wird. Das eine werden jedenfalls viele Praktiker zugeben, daß der unterernährte Diabetiker die „Gewöhnung“ an eine niedrige Kalorienzufuhr auffallend häufig zeigt.

Auf die Fettsucht will ich hier nicht eingehen, da v. Bergmann auf Grund sehr interessanter Untersuchungen darüber spreclen wird.

Wenn wir die übrigen Krankheiten betrachten, so sind es namentlich die Gicht und der Diabetes mellitus, die eine Verlangsamung einzelner Stoffwechselprozesse beobachten lassen, also diejenigen Krankheiten, die schon Bouchard ${ }^{4}$ ) zusammen mit der Fettsucht, aber freilich mit noch vielen anderen pathologischen Zuständen und auf Grund absolut ungenügender Buweise unter dem Namen „Maladies par ralentissement de la nutrition" zusammenfaßte. Ein besonderes Interesse gewinnt diese Tatsache dadurch, daß wir eine Verlangsamung des Gesamtstoff wechsels, eine Herabsetzung des Energieumsatzes auch beim Ausfall der Schilddrüsen. funktion beobachten. Dem wäre noch der Ausfall einer an. dern Drüse ohne Ausführungsgang, des 0 variums, anzureihen, der, wie Loewy und Richter ${ }^{5}$ ) und neuerdings L. Zuntz ${ }^{5}$ ) be-

1) Die Gesetze des Energieverbrauchs bei der Ernăhrung. S. 269 ff., 324. - 2) Hof. meisters Beitrăge zur chemischen Physiologie und Pathologie, Bd. 9, S. 333. - 3) Pflügers Archiv, Bd. 109, S. 473. - 4) Zeitschrift für klinische Medizin, Bd. 43, S. 86. - 5) Malađie par ralentissement de la nutrition. Paris 1890 . - 6) Archiv für Physiologie 1899. Suppl. - Deutsche Zeitschrift für Chirurgie, Bd. 95 , S. 250. 
wiesen haben, wenigstens bisweilen zu einer Herabsetzung des Gesamtumsatzes führt.

Sie sehen also, daß eine Verlangsamung des Stoffwechsels doch nicht so selten ist, wie man geneigt ist anzunehmen, und dal sie in mehrfacher Hinsicht unsere Aufmerksamkeit verdient. Von großem theoretischem Interesse ist die Tatsache, daß eine Verlangsamung von Stoffwechselvorgängen einerseits bei den sogenannten Stoffwechselkrankheiten, anderseits bei Ausfall der Funktion von Drüsen mit innerer Sekretion vorkommt. Das läbt hoffen, dal die genauere Untersuchung des Einflusses dieser Organe auf den zeitlichen Ablauf der Zer. setzungen, wie sie namentlich Falta, Eppinger und Rüding $\operatorname{gr}^{1}$ ) unternommen haben, auch das Verständnis der Stoffwechselkrankheiten fördern wird.

Aber auch ein direkt praktisches Interesse bieten einzelne der besprochenen Tatsachen. Erstens ist die Kenntnis von der Verlangsamung des Nukleïnstoffwechsels bei der Gicht ausschlaggebend für die Behandlung, und zweitens wird die Feststellung, daß viele Diabetiker eine Anpassung an verminderte Nahrungszufuhr zeigen, unsere Scheu vor der Unterernährung Zuckerkranker überwinden und uns in sehweren Fällen einen Versuch mit Herabsetzung der Eiweißzufuhr wagen lassen, auch wenn dabei die Kalorienzufuhr vorübergehend ungenügend wird. 